九州大学学術情報リポジトリ

Kyushu University Institutional Repository

\title{
WEAK PARETO OPTIMALITY OF MULTIOBJECTIVE PROBLEM IN A BANACH SPACE
}

Minami, Masayoshi

Associate Professor, Department of Management, Faculty of Economics, Yamaguchi University

https://doi.org/10.5109/13145

出版情報: 統計数理研究. 19 (3/4), pp. 19-23，1981-03. Research Association of Statistical Sciences

バージョン :

権利関係 : 


\title{
WEAK PARETO OPTIMALITY OF MULTIOBJECTIVE PROBLEM IN A BANACH SPACE
}

\author{
By \\ Masayoshi Minami* \\ (Received August 10, 1980)
}

\section{Introduction.}

In the previous paper ([5]), we studied the ordinary multiobjective convex program on a locally convex linear topological space in the case that the objective functions and the constraint functions were continuous and convex, but not always Gâteaux differentiable. In the case, we showed that the generalized Kuhn-Tucker conditions given by a subdifferential formula were necessary and sufficient for weak Pareto optimum.

In this paper, we consider the ordinary multiobjective program on a Banach space in the case that objective functions and constraint functions are locally Lipschitzian but not always convex, and derive Kuhn-Tucker forms given by Clarke's generalized gradients ([1]) as necessary conditions for weak Pareto optimum. Theorem 2.1 is a generalization of Theorem 1.1 of Schechter ([6]) which is concerned to ordinary program with a scalar-valued objective function.

In this paper, $X$ and $X^{*}$ are a real Banach space and its continuous dual, whose origins are denoted by $\theta$ and $\theta^{*}$, respectively. By $\varnothing$ we denote the empty set.

\section{Necessary condition for weak Pareto optimality.}

Let $f_{1}, f_{2}, \cdots, f_{m}, g_{1}, g_{2}, \cdots, g_{n}$ be real-valued functions on a real Banach space $X$ and $A$ be a subset of $X$. We denote $\Omega_{j}=\left\{x \in X: g_{j}(x) \leqq 0\right\}, j=1,2, \cdots, n$ and $\Omega=\bigcap_{j=1}^{n} \Omega_{j} \cap \Lambda$.

A vector $x_{0} \in \Omega$ is called to be weak Pareto optimal if there is no $x \in \Omega$ such that $f_{i}(x)<f_{i}\left(x_{0}\right)$ for every $i=1,2, \cdots, m$.

The ordinary multiobjective program is the problem to find vectors $x_{0} \in \Omega$ of weak Pareto optimum and written by $(\Omega, f)$, where $f=\left(f_{1}, f_{2}, \cdots, f_{m}\right)$.

A real-valued function $f$ on $X$ is said to be locally Lipschitzian if any point in $X$ admits a neighborhood $U$ such that, for some constant $K$, for all $y$ and $z$ in $U$, we have

* Associate Professor, Department of Management, Faculty of Economics, Yamaguchi University Yoshida, Yamaguchi, Japan. 


$$
|f(y)-f(z)| \leqq K\|y-z\|,
$$

where $\|x\|$ denotes the norm of $x$.

For a locally Lipschitz function $f$ and for $x_{0}, x$ in $X, f^{\circ}\left(x_{0} ; x\right)$ defined as follows is said to be the generalized directional derivative in the direction $x$ at $x_{0}$;

$$
f^{\circ}\left(x_{0} ; x\right)=\lim _{\substack{h \rightarrow \theta \\ t \vee 0}} \frac{f\left(x_{0}+h+t x\right)-f\left(x_{0}+h\right)}{t} .
$$

We denote by $\partial^{*} f\left(x_{0}\right)$ the subdifferential of the convex continuous function $f^{\circ}\left(x_{0} ; \cdot\right)$ at $\theta$, that is,

$$
\partial^{*} f\left(x_{0}\right)=\left\{\phi \in X^{*}: f^{\circ}\left(x_{0} ; x\right) \geqq \phi(x) \text { for every } x \in X\right\} .
$$

We call it Clarke gradient of $f$ at $x_{0}([1])$.

By the normal cone of convex set $A$ at the point $x_{0}$ in $\Lambda$ we mean the set

$$
\left\{\phi \in X^{*}: \phi\left(z-x_{0}\right) \leqq 0 \text { for every } z \in \Lambda\right\}
$$

and denote it by $N\left(x_{0}, A\right)([4])$.

The main theorem is the following:

THEOREM 2.1. Assume that all objective functions $f_{i}, i=1,2, \cdots, m$ and all constraint functions $g_{j}, j=1,2, \cdots, n$, are locally Lipschitzian and that the constraint set $\Lambda$ is a convex subset of $X$. Then, if $x_{0}$ is weak Pareto optimal for the ordinary multiobjective program $(\Omega, f)$, there exist some real numbers $\lambda_{i} \geqq 0(i=1,2, \cdots, m), \mu_{j} \geqq 0$ $(j=1,2, \cdots, n)$ with $\left(\lambda_{1}, \cdots, \lambda_{m}, \mu_{1}, \cdots, \mu_{n}\right) \neq(0, \cdots, 0)$ and some vectors $y_{i} \in \partial^{*} f_{i}\left(x_{0}\right)$ $(i=1,2, \cdots, m), z_{j} \in \partial^{*} g_{j}\left(x_{0}\right)(j=1,2, \cdots, n), w \in N\left(x_{0}, \Lambda\right)$ such that

$$
\sum_{i=1}^{m} \lambda_{i} y_{i}+\sum_{j=1}^{n} \mu_{j} z_{j}+w=\theta^{*}
$$

and $\mu_{j} g_{j}\left(x_{0}\right)=0(j=1,2, \cdots, n)$.

\section{Proof of Theorem 2.1.}

In this section, we recall a few definitions and state lemmas which we use to prove Theorem 2.1 .

Definition 3.1. Let $f: X \rightarrow R^{1}$ and $x_{0} \in X$. A vector $\bar{x} \in X$ is called a direction of decrease of $f$ at $x_{0}$ if there exist a neighborhood $V$ of the vector $\bar{x}$ and a real $\varepsilon>0$ such that $x \in V$ and $0<t<\varepsilon$ imply $f\left(x_{0}+t x\right)<f\left(x_{0}\right)$. The set of all such vectors $\bar{x}$ is denoted by $D C\left(x_{0}, f\right)$.

REMARK 3.1. The set $D C\left(x_{0}, f\right)$ is an open cone with vertex at the origin $\theta$ in $X$, or else it is void.

Definition 3.2. Let $\Omega$ be a subset of $X$ and let $x_{0} \in X$. A vector $\bar{x} \in X$ is called an admissible direction with respect to $\Omega$ at $x_{0}$ if there exist a neighborhood $V$ of the vector $\bar{x}$ and a real $\varepsilon>0$ such that $x \in V$ and $0<t<\varepsilon$ imply $x_{0}+t x \in \Omega$. The set of all such vectors $\bar{x}$ is denoted by $A C\left(x_{0}, \Omega\right)$.

REMARK 3.2. The set $A C\left(x_{0}, \Omega\right)$ is an open cone with vertex at the origin $\theta$ in $X$.

Definition 3.3. Let $A$ be a subset of $X$ and let $x_{0} \in X$. A vector $\bar{x} \in X$ is called 
a tangent direction to $A$ at $x_{0}$ if there exist a real $\varepsilon>0$ and a mapping $r:[0, \varepsilon] \rightarrow X$ such that $x_{0}+t \bar{x}+r(t) \in \Lambda$ for all $t$ in $[0, \varepsilon]$ and $r(t) / t \rightarrow \theta$ as $t \downarrow 0$ where $[0, \varepsilon]$ denotes the closed interval $0 \leqq t \leqq \varepsilon$. The set of all such vectors $\bar{x}$ is denoted by $\operatorname{TC}\left(x_{0}, A\right)$.

REMARK 3.3. The set $T C\left(x_{0}, \Lambda\right)$ is a cone with vertex at the origin $\theta$ in $X$.

Definition 3.4. The negative polar cone of a cone $K$ with vertex at the origin $\theta$ in $X$ is the set

$$
K^{\circ}=\left\{\phi \in X^{*}: \phi(x) \leqq 0, x \in K\right\} .
$$

Lemma 3.1. ([4]). Let $g: X \rightarrow R^{1}$ be any function and $\Omega=\{x \in X: g(x) \leqq 0\}$. Then, if $g\left(x_{0}\right)=0, D C\left(x_{0}, g\right) \subset A C\left(x_{0}, \Omega\right)$.

LEMmA 3.2. ([6]). Let $f: X \rightarrow R^{1}$ be a locally Lipschitz function and $x_{0} \in X$. If $f^{\circ}\left(x_{0} ; \bar{x}\right)<0$, then $\bar{x} \in D C\left(x_{0}, f\right)$.

Lemma 3.3. ([6]). Let $f: X \rightarrow R^{1}$ be a locally Lipschitz function and $x_{0} \in X$. Define $C=\left\{x \in X: f^{\circ}\left(x_{0} ; x\right)<0\right\}$ and assume $\theta^{*} \in \partial^{*} f\left(x_{0}\right)$, then $C^{\circ}=\left\{\lambda y: \lambda \geqq 0, y \in \partial^{*} f\left(x_{0}\right)\right\}$.

LEMmA 3.4. (Dubovitskii-Milyutin, [2]-[4]). Let $K_{0}$ be a convex cone with vertex at the origin $\theta$ of $X$ and $K_{1}, \cdots, K_{n}$ open convex cones with vertex at the origin $\theta$ of $X$. Then $\bigcap_{i=0}^{n} K_{i}=\varnothing$ if and only if there exist some $y_{i}^{*} \in K_{i}^{\circ} i=0,1, \cdots, n$, not all identically $\theta^{*}$, such that

$$
y_{0}^{*}+y_{1}^{*}+\cdots+y_{n}^{*}=\theta^{*} .
$$

Lemma 3.5. ([4]). For a subset $A$ of $X$ and $x_{0} \in X$, define $K_{0}=T C\left(x_{0}, A\right)$. Then, if $\Lambda$ is a convex subset of $X, K_{0}^{\circ}=N\left(x_{0}, A\right)$.

LEMMA 3.6. Uuder the assumption on Theorem 2.1, define $K_{0}=T C\left(x_{0}, \Lambda\right)$,

$$
K_{j}=\left\{x \in X: g_{j}^{\circ}\left(x_{0} ; x\right)<0\right\}, \quad j=1,2, \cdots, n
$$

and

$$
K_{n+i}=\left\{x \in X: f_{i}^{\circ}\left(x_{0} ; x\right)<0\right\}, \quad i=1,2, \cdots, m,
$$

then $K_{0}$ is a convex cone at $\theta$ and $K_{i}$ are open convex cones at $\theta$ for $i=1,2, \cdots, n+m$. If $x_{0}$ is weak Pareto optimal for the ordinary multiobjective program $(\Omega, f)$, then there exist some $y_{i}^{*} \in K_{i}^{\circ}$ for $i$ in $\{0, n+1, \cdots, n+m\} \cup J$, not all identically $\theta^{*}$, such that

$$
y_{0}^{*}+\sum_{j \in J} y_{j}^{*}+\sum_{i=1}^{m} y_{n+i}^{*}=\theta^{*}
$$

where $J=\left\{j \in N: g_{j}\left(x_{0}\right)=0\right\}$ and $N=\{1,2, \cdots, n\}$.

ProOF of LEMMA 3.6. By Lemma 3.4, it is sufficient to prove that

$$
K_{0} \cap\left(\bigcap_{j \in J} K_{j}\right) \cap\left(\bigcap_{i=1}^{m} K_{n+i}\right)=\varnothing
$$

holds. Suppose the contrary that there exists some

$$
\bar{x} \in K_{0} \cap\left(\bigcap_{j \in J} K_{j}\right) \cap\left(\bigcap_{i=1}^{m} K_{n+i}\right) .
$$

By Lemma 3.1 and Lemma 3.2,

$$
\bar{x} \in D C\left(x_{0}, f_{i}\right) \quad \text { for each } i=1,2, \cdots, m,
$$


and

$$
\bar{x} \in D C\left(x_{0}, g_{j}\right) \subset A C\left(x_{0}, \Omega_{j}\right) \quad \text { for each } j \in J .
$$

Then there exist a sufficiently small $t_{1}>0$ and some vector $x_{1}$ in a neighborhood of $\bar{x}$ such that

and

$$
\begin{array}{ll}
f_{i}\left(x_{0}+t_{1} x_{1}\right)<f_{i}\left(x_{0}\right) & \text { for every } i=1,2, \cdots, m, \\
x_{0}+t_{1} x_{1} \in \Omega_{j} & \text { for every } j \in J, \\
x_{0}+t_{1} x_{1} \in \Lambda &
\end{array}
$$

$$
x_{0}+t_{1} x_{1} \in \Omega_{j} \quad \text { for every } \quad j \in N-J .
$$

The last follows from $N-J=\left\{j \in N: g_{j}\left(x_{0}\right)<0\right\}$ and from that $x_{0}$ is an interior point of a subset $\Omega_{j}$ in $X$ for each $j \in N-J$. This contradicts the weak Pareto optimality of $x_{0}$. Hence we have the conclusion of Lemma 3.6.

Proof of THEOREM 2.1. Let $x_{0}$ be weak Pareto optimal. In the case that $\theta^{*} \notin \partial^{*} f_{i}\left(x_{0}\right)$ for every $i=1,2, \cdots, m$ and that $\theta^{*} \notin \partial^{*} g_{j}\left(x_{0}\right)$ for every $j$ in $J$, by Lemma 3.6 there exist some $y_{i}^{*} \in K_{i}^{\circ}$ for $i$ in $\{0, n+1, \cdots, n+m\} \cup J$, not all identically $\theta^{*}$, such that

$$
y_{0}^{*}+\sum_{j \in J} y_{j}^{*}+\sum_{i=1}^{m} y_{n+i}^{*}=\theta^{*} .
$$

Furthermore, by Lemma 3.3 and Lemma 3.5 we have

$$
\begin{array}{ll}
K_{n+i}^{\circ}=\left\{\lambda y: \lambda \geqq 0, y \in \partial^{*} f_{i}\left(x_{0}\right)\right\} & \text { for } i=1,2, \cdots, m, \\
K_{j}^{\circ}=\left\{\mu z: \mu \geqq 0, z \in \partial^{*} g_{j}\left(x_{0}\right)\right\} & \text { for } j \text { in } J
\end{array}
$$

and $K_{0}^{\circ}=N\left(x_{0}, \Lambda\right)$. For $j$ in $N-J$, put $\mu_{j}=0$, then we have the conclusion of Theorem 2.1 except the condition $\left(\lambda_{1}, \cdots, \lambda_{m}, \mu_{1}, \cdots, \mu_{n}\right) \neq(0, \cdots, 0)$. Supposed the contrary that $\left(\lambda_{1}, \cdots, \lambda_{m}, \mu_{1}, \cdots, \mu_{n}\right)=(0, \cdots, 0)$, then $y_{i}^{*}=\theta^{*}$ for $i \in\{0, n+1, \cdots, n+m\} \cup J$ which is the contradiction.

In the case that $\theta^{*} \in \partial^{*} f_{i_{0}}\left(x_{0}\right)$ for some $i_{0}$, sufficiently let $\lambda_{i_{0}}$ be any positive number, $\lambda_{i}=0$ except for $i=i_{0}, \mu_{j}=0$ for $j=1,2, \cdots, n, y_{i_{0}}=\theta^{*}, y_{i}$ be any vector in $\partial^{*} f_{i}\left(x_{0}\right)$ except for $i=i_{0}, z_{j}$ be any vector in $\partial^{*} g_{j}\left(x_{0}\right)$ for $j=1,2, \cdots, n$ and $w=\theta^{*}$.

In the other case that $\theta^{*} \in \partial^{*} g_{j_{0}}\left(x_{0}\right)$ for some $j_{0}$ in $J$, sufficiently let $\lambda_{i}=0$ for $i=1,2, \cdots, m, \mu_{j_{0}}$ be any positive number, $\mu_{j}=0$ except for $j=j_{0}, y_{i}$ be any vector in $\partial^{*} f_{i}\left(x_{0}\right)$ for $i=1,2, \cdots, m, z_{j_{0}}=\theta^{*}, z_{j}$ be any vector in $\partial^{*} g_{j}\left(x_{0}\right)$ except for $j=j_{0}$ and $w=\theta^{*}$.

\section{References}

[1] Clarke, F.H., A new approach to Lagrange multipliers, Mathematics of Operations Research, 1 (1976), 165-174.

[2] Dubovitskit, A. YA. and Milyutin, A.A., Extremum problems in the presence of restrictions, USSR Computational Methematics and Mathematical Physics, 5 (1965), 1-80.

[3] Girsanov, I. V., Lectures on Mathematical Theory of Extremum Problems, Springer- 
Verlag, Berlin •Heidelberg $\cdot$ New York, 1972.

4] Holves, R.B., A Course on Optimization and Best Approximation, Springer-Verlag, Berlin·Heidelberg $\cdot$ New York, 1972.

[5]- Misam, M., Weak Pareto optimality of multiobjective problems in a locally convex linear topological space, J. Optimization Theory and Applications, 34 (1981), to appear.

[6] Schechter, M., More on subgradient duality, J. Mathematical Analysis and Applications, 71 (1979), 251-262. 